oxyd aus der Gewichtsdifferenz zu rechnen. In diesem Falle ist das beschriebene Verfahren noch viel kürzer als das von A $\mathrm{n} g$ e $\mathrm{n}$ o $\mathrm{t}$ angegebene.

\section{Zur Untersuchung von Formaldehydpastillen.}

\section{Von Dr. ERnst Rüst, St. Gallen, Schweiz.} (Eingeg. d. 10.2. 1906.)

Das Trioxymethylen oder der ,feste Formaldehyd" wird in der Desinfektionspraxis in immer steigendem Maße verwendet. Es empfiehlt sich Jeswegen, die verschiedenen im Handel befindlichen Produkte auf ihre Eignung zu genanntem Zwecke näher zu untersuchen. Bei der Wertbestimmung von Formaldehydpastillen kommt nicht allein der Gehalt an Formaldehyd in Betracht, sondern namentlich auch die Kohleabscheidung, die beim Verflüchtigen in der Wärme stattfindet. Manche Sorten Pastillen geben einen starken kohligen Rückstand, der am Boden des Verdampfungsgefäßes eine schlecht leitende Schicht bildet und verhindert, daß die Pastillen in der berechneten Zeit und mit der berechneten Menge Brennmaterial vollständig verdampfen, abgesehen davon, daß durch die zurückgebliebenen Produkte der Apparat stark verunreinigt wird. Für die praktische Untersuchung der Pastillen empfiehlt es sich, nach den bis jetzt gemachten Erfahrungen folgende Werte zu bestimmen :

1. Gewicht der Pastillen. Eine größere Anzahl Pastillen wird gewogen und der Mittelwert berechnet. Die Pastillen sollen möglichst genau $1 \mathrm{~g}$ wiegen. Da sie beim praktischen Gebrauche abgezählt werden; so wird bei leichteren Pastillen zu wenig Formaldehyd entwickelt, während bei schwereren Tabletten ein unnützer Mehrverbrauch des nicht eben billigen Materials stattfindet.

2. Löslichkeit in hei Bem Wasser. $\mathrm{Zu}$ einer fein gepulverten Pastille wird ca. $100 \mathrm{ccm}$ Wasser gegeben, dann wird die Lösung zum Kochen erhitzt und einige Zeit im Sieden erhalten. Gute Pastillen hinterlassen keinen Rückstand oder nur wenige weiße Flocken.

3. Reaktion der wässerigen Lösung. Die erhaltene Lösung wird mit einem Tropfen einer Phenolphtaleïnlösung versetzt; falls sie sich dabei nicht rötet, gibt man einen Tropfen $1 / 10^{-n}$. Alkali zu. Tritt jetzt Rotfärbung auf, so sind die Pastillen neutral. Technisch reines Trioxymethylen reagiert neutral. Bei Anwesenheit von Alkali oder Säure müssen diese zur folgenden Formaldehydbestimmung titrimetrisch bestimmt werden.

4. Formaldehyd. Diese Bestimmung wird am besten nach der von $B \mathrm{l}$ a $\mathrm{n} \mathrm{k}$ und $\mathrm{Fin}$ ken beiner angegebenen und vom Verf. modifizierten Metode ausgeführt1). Ein gutes Produkt zoigt $95 \%-97 \%$ Formaldehyd; es gibt aber im Handel so che mit nur $85 \%$ Gehalt.

1) Diese $Z \quad 19,138$ (1906)
5. Kohliger Rüekstand. In einer kleinen Platinschale werden 10 gewogene Pastillen mit dem Brenner so weit erhitzt, daß sie zu brennen anfangen. Dann läßt man sie ohne weitere Wärmezufuhr ruhig von selbst abbrennen. Der Rückstand wird gewogen. Brauchbare Pastillen sollen bei dieser Methode nicht viel über 0,1\% Rückstand hinterlassen; solche mit $0,5 \%$ sind ganz unbrauchbar. Beim Verdampfen der Tabletten im Desinfektionsapparat findet allerdings keine Verbrennung statt; es hat sich aber gezeigt, daß mit dieser Schnellmethode Werte erhalten werden, die mit den in der Praxis auftretenden Rückständen parallel gehen.

6. A s c hengeh a l t. Die unter 5. erhaltene Kohle wird verascht. Die Asche soll nicht mehr als $0,05-0,08 \%$ betragen. Die Reaktion sei neutral oder ganz schwach alkalisch.

Es ist auch empfehlenswert, auf die bei der Behandlung unter 5. auftretenden Verbrennungsgase zu achten. Bei der Untersuchung einer Anzahl Pastillensorten ist uns ein Produkt begegnet, das beim Verbrennen stark saure und nach Chlor riechende Gase, die Jodkaliumstärkepapier bläuten, entwickelte. Bei der Formaldehydbestimmung trat ein chloroformähnlicher Geruch auf.

Chemisches Laboratorium des Kantons St. Gallen.

\section{Das Niedenfïhrsche Intensivsystem.} II.

\author{
Von Dr: M. Neduans-Cronberg i. T. \\ (Eingeg. d. 30.4. 1900?)
}

Ich habe Direktor Fritz L ütys Artikel resp. Vortrag (diese Z. 18, $1253 \mathrm{ff}$. [1905]) zum Gegenstand einer Kritik gemacht und hätte demnach von ihm, dem angegriffenen Autor, eine Entgegnung erwarten dürfen. Statt seiner antwortet H. H. N i e d e $\mathrm{n} f \ddot{u} \mathrm{~h}$ r. Damit ist aber die Frace gerechtfertigt, warum dieser sich von der für alle Wissenschaften üblichen Gepflogenheit emanzipiert, selber die publizistischen Besprechungen seiner Entdeckungen und Erfindungen zu übernehmen, und aus welchen Gründen er sie jemand übertragen hat, der sich in der Hauptsache auf die ihm gemachten Mitteilungen verlassen mußte. Inwieweit ein solches Verfahren geeignet ist, für kommende Fälle als unliebsamer Präzedenzfall zu dienen, überlasse ich der allgemeinen Beurteilungl).

So notwendig es war, diesen Punkt zur Sprache zu bringen, ich habe es ungern getan und würde es nicht getan haben, wenn $\mathrm{H} . \mathrm{H}, \mathrm{N}$ i e d e $\mathrm{n} f \ddot{\mathrm{u} h \mathrm{r}}$ in seiner Entgegniung (diese Z. 19, $61 \mathrm{ff.} \mathrm{[1906])}$ mich nicht auf das allerschwerste provoziert, wenn er mir nicht die Vorwürfe der Unśachlichkeit und der persönlichen Animosität gemacht und sich nicht gescheut hätte, sich in eine psychologische Unter-

1) Wir muBten Herrn Dr. Neumann dis Wort auch zu einigen persönlichen Bemerkungen geken, bitten jedoch die Delatte von nun an im rein sachlichen Fahrwasser zu halten.

Die Rrdaktion. 
suchung über die Motive einzulassen, die mich zu meinen kritischen Bemerkungen veranlaßt haben.

Zur Steuer der Wahrheit muß ich ihm deshalb die Versicherung geben, daß es zum mindesten vier Beweggründe gewesen, die meine Bedenken überwunden, mich direkt oder indirekt mit ihm in einen literarischen Streit einzulassen, von denen der kleinste nicht der war, der im obigen des näheren auseinandergesetzt wurde. Inwieweit sein Vorwurf zutrifft, daß in einem Einspruche seinerseits - um nicht den Eindruck zu erwecken, als ob dieser Einspruch Erfolg gehabt, wäro in loyaler Weise besser zu sagen gewesen, in der Einleitung des Beschwerdeverfahrens ${ }^{2}$ ), - gegen eine meinerPatentanmeldungen die Veranlassung zu meiner Abhandlung zu suchen sei, möge aus der Tatsache erhellen, $d$ a $B$ i $\mathrm{ch}$ jetzt erst von H. H. Niedenf ühr höre, da $B$ er auch in diesem Falle fürgut befunden, sich selbstals Interes. senten $z u$ decken und jemand an. ders als Einsprechenden etc. für sich vorzuschieben.

Ich bedaure lebhaft, daß es $\mathrm{N}$ i ed en $\mathrm{füh} \mathrm{r}$ nicht möglich gewesen, auf meine streng sachlichen Angriffe sachlich zu antworten. Derartige Mittel, wie er sie gebraucht, dem Gegner die bona fides abzusprechen, sind auf wissenschaftlichem Gebiete ungewohnte Maßnahmen; selbst im struggle for life dürfte nur in Ausnahmefällen von Liebhabern zu ihnen gegriffen werden, und dann auch wohl nur, wenn in Ermangelung sachlicher Einwände weiter nichts zu sagen, oder wenn die Position so gefährdet erscheint, daß eben nicht mehr viel zu verlieren ist. Und was tun hier schließlich die Motive? Nicht sie sind maßgebend, sondern lediglich die Beweiskraft der gegen sein System vorgebrachten Argumente.

Ich beklage diese Art der Polemik; die Anerkennung wird man mir indes nicht versagen können, daß mein erster Artikel in keiner Weise Grund zu derartigen, persönlichen Ausfällen gegeben, und daß ich mich jetzt im Zustande der legitimen $A b$ wehr befinde.

Wie schon L ü ty s Ausführungen Próf. Dr. $\mathrm{D} u$ is b e $\mathrm{r}$ V Veranlassung gaben zu konstatieren, da $\mathrm{B}$ die Einführung des Ventilators nicht $\mathrm{N}$ i e. d e $n f u ̈ h r s$ Verdienst sei, so veranlaßten sie mich, diesen Faden weiter auszuspinnen, um der Legendenbildung vorzubeugen, als ob das Prinzip des Überdrucks in den Kammern, als ob die Zweiteilung des Glovers usw. $\mathrm{N}$ i ed e $\mathrm{n} f \ddot{\mathrm{u}} \boldsymbol{h} \mathbf{r}$ zuge. schrieben werden müsse.

L ü t y sagt wörtlich (diese Z. 18, 1256 [1905]): „Das D. R. P. hat nun dieselben in einfachster und erfolgreichster Weise beseitigt, indem $H$. $H$. $\mathrm{N}$ i ed en $f$ ühr, der Erfinder desselben, di e Funktionen des Gloverapparates a uf $\mathrm{z}$ wei Türme verteilte und den Zugerreger zwischen beide plazierte". Außerdem ist zu lesen: „So stehen wir gegenwärtig mit den Erfolgen des Verfahrensdes D. R. P. $140825 R$ esultaten ge genü ber, die für die gesamte chemische Industrie vongrößter Bedeutung sind". Er sagt

2) Durch inzwischen erfolgte Patenterteilung ist auch das Bischwerdoverfahren zu Ungunsten Nid:nführs entschieden worden. weiterhin S. 1261: ,,ein $\mathrm{K}$ a m mersystem, nach Niedenf $\ddot{u}$ hrs Grundsätzen ausgeführt", das im übrigen ,,nicht nur erlaubt, schon existierende Systeme jeglicher Ausführungsweise mit minimalen Unkosten lukrativer zu machen, sondern auch die fehlerhaften wieder in Ordnung zu bringen".

Ich denke, das ist deutlich genug, und wenn $\mathrm{N}$ i e d e $\mathrm{n} f \ddot{\mathrm{u}} \mathrm{h} \mathrm{r}$ in seiner Bescheidenheit, der Ansicht ist, daß sie übertrieben waren, so hätte er mit einen Äußerungen, mir ,beliebe es, ihm maßlose Selbstüberhebung anzudichten ", und ich ,erlaube mir, ihm Anmaßung unterzulegen", sich nicht an meine, sondern an $\mathrm{L} \ddot{\mathrm{u}} \mathrm{t}$ y s Adresse wenden sollen. Da er das nicht tat, hatte ich das Recht anzunehmen, er sei mit ihnen einverstanden.

H. H. Niedenf ührs Bescheidenheit ist denn wirklich auch nur zu berechtigt, wenn er zugibt, seine Erfindung resp. sein System sei nichts als eine ,Schlußfolgerung ans Ideen und Erfahrungen, die der Praxis der Schwefelsäureindustrie usw. ", $d$. h. nichts als eine Kombination von an sich bekannten, erprobten Einzelheiten, die zu benutzen jedermann freisteht, um in der von mir angegebenen Weise erforderlichenfalls auf dieselbe Art zu arbeiten wie H. H. N i e d e $\mathrm{n} f \ddot{u} \mathrm{~h}$ r. Um über diese Einzelheiten ein klares Bild zu bekommen und um keinen Zweifel zu lassen, wem ihre Priorität gebührt, sei es gestattet sie aufzuzählen :

1. Idee der Intensivproduktion: Franzosen, besonders $\mathrm{B}$ en $\mathrm{k}$ er.

2. Ventilator resp. Doppelventilator: Mulden $\mathrm{h}$ ü t te und F alding.

3. Doppelglover: $\mathrm{F}$ a I d i n g.

4. Obere Gaseinführung: T h. M e yer.

5. Überdruck in den Kammern: vo n jeher.

6. Hohe Kammern: B enker.

7. Plattenturm: I u n g e.

8. Wassereinspritzung: S prengel.

9. Gasreinigung: $\mathrm{R} a \mathrm{th}$, resp. Kontaktprozesse.

Es ist mir nicht beigefallen, das Fal ding sche D. R. P. 76691 und das Z $\mathrm{Z}$ nner sche D. R. P. 134661 mit dem D. R. P. 140825 identifizieren zu wollen; ist doch in jenen beiden Verfahren mit keinem Wort von Ventilatoren die Rede. Ich habe lediglich gezeigt, da $B$ ihre Kombination mit Ven tilatoren indenstandsetzen wïrde, unter analogen $B$ edingungen zuarbeiten als nach dem Verfahrendes D. R. P. 140825 , unter der Voraussetzung, daß der zweite Glover resp. $Z$ a n n e r s Glover eben voller Glover und nicht lediglich Denitrifikator ist. In $\mathrm{F}$ a Id i ngs Konzentrator - gleichgültig, ob Säure von 65,63 oder $61^{\circ}$ Bé. erzielt wird, je nach der Menge der aufgegebenen Säure zur Größe des Systems - werden ebenso die Verunreinigungen zurückgehalten, werden in ähnlicher Weise die Gase gekühlt wie in $\mathbf{N}$ i e $d$ e $\mathbf{n} f$ üh r s Konzentrationsglover. Anstatt mit $70-80^{\circ}$ wie bei $\mathrm{Nie}$. denführ würden die Gase hier mit etwa $150-180^{\circ}$ in den 2. Turm treten können, die nach Bod e (L unge I., 588 [1903]) vollkommen genügen, um volle Gloverwirkung $\mathrm{zu}$ er- 
zielen. Der Vorteil dor Salpetersäureersparnis in dem von $\mathrm{N}$ ied e $\mathrm{n} f \mathrm{u} h \mathrm{~h}$ und mir (Pat.-Anm. $\mathrm{Nr} .6190$ ) vorgeschlagenen Sinne würde auch in diesem Falle zutreffen müssen, da bei Temperaturen von $150 \_180^{\circ}$ ebenso wie bei jenen von $70-80^{\circ}$ jede Reduktion der Stickoxyde zu $\mathrm{N}_{2} \mathrm{O}$ und $\mathrm{N}$ ausgeschlossen sind. Weiterhin wird ge zeigt werden, daßsehon vom theo. retischen Standpunkt a s ein der. artiges Arbeiten erheblich empfehlenswerter und ausichtsreicher sein muB, als der Niedenführsehe Vorschlag.

$\mathrm{N}$ i ed e $\mathrm{n} f$ ü h $\mathrm{r}$ will dem Leser zur Beurteilung überlassen, in wieweit meine Behauptung berechtigt sei, daß der Einfluß der Verunreinigungen der Röstgase für den Salpetersäureverbrauch eines Kammersystems als ,nicht ins Gewicht fallend“" bezeichnet werden müsse. Ich bin ganz damit einverstanden, möchte aber nicht verfehlen darauf hinzuweisen, daß ich doutlich gesagt: „Auch trotz der Staubsammler, Staubkammern, Gasreinigung usw.". Die noch mit hinübergerissenen geringen Mengen Flugstaub werden wie sonst in dem oder den Glovern niedergeschlagen und kommen in beiden mit nitrosen Gasen und Flüssigkeiten in Berührung -im ersten allerdings mit schwächeren ${ }^{3}$ ) und nach ihrer weiteren Elimination deren letzten Reste im zweiten mit stark nitrosen. Die Unvollständigkeit der Fernhaltung des Flugstaubs von der Nitrose auf Grund des Verfahrens des D. R. P. 140825 gibt $\mathrm{Ni}$ ed en $\mathrm{f} \ddot{\mathrm{u}} \mathrm{hr}$ übrigens selber zu, durch seine neueste Anmeldung Nr. 7013, in der er die den 1. Glover bereits passierten Flugstaubmengen, bevor sie in den Denitrierglover treten, durch aus den Kontaktprozessen bekannte Gasfilter usw, zurückhalten will. Wie weiter unten erwiesen werden wird, bedingt aber die Praxis des $\mathrm{N}$ i e d e $\mathbf{n} \mathbf{f} \ddot{u} \mathrm{~h} \mathrm{r}$ schen Systems eine wiederholte Anwendung der zwischen Glover und Gay-Lussac zirkulierenden Berieselungssäure, die durch Konzentration eines nur sehr geringen Teils Kammersäure eine nur recht begrenzte Verjüngung erfährt und die an Verunreinigungen aller Art sich immer mehr anreichern muß. Ni ed en fü hr bringt demnach auch im zweiten Glover immer mehr arsenige Säure in Berührung mit der Nitrose, so daß in dieser Beziehung gegenüber diesem offenbaren Nachteil der angegebene Vorteil der ,,vorgereinigten Gase" als nicht recht verständlich bezeichnet werden muß. Schon aus diesem Grunde ist die dem ersten Glover folgende Gasreinigung als verfehlt zu kennzeichnen.

Seine diesbezüglichen Angriffe gegen mich stïtzt er offenbar auf die in jener Beschreibung (P. A. Nr. 7013) geäuBerten Behauptungen, ,daB das Arsen aus den Schwefligsäuregasen in einem Stadium des Verfah. rens entfernt werde, in welchem die Einwirkung von Salpetersäure noch nicht stattfindet," und ,da bei den bisherigen Verfahren zur Darstellung von Schwefelsäure das Arsen selbst durch die Einwir-

${ }^{3}$ ) In wieweit $L$ ü t y s resp. N i e d e $n$ f ü h r s Behauptungen zutreffend sind, diese Säure enthalte nur ca. $0,2 \% 36^{\circ}$ iger Salpetersäure, wird weiter unten erörtert werden. kung von Salpetersäure zu Arsensäure oxydiert wurde, so wurde eine erhebliche Menge von Salpetersäure stets nutzlos versehwendet". In dieser Form ist nicht nur die erste Belıuptung grundfalsch, wic erwiesen, sondern auch die zweite. Im Glover und in den Kammern kann bekanntlich die Oxydationswirkung der Salpetersäure auf arsenige Säure wegen der entgegenstehenden Reduktionswirkung der schwefligen Säure eine nur recht geringe sein. Dis hierbei in derselben Weise wie bei der Oxydation der schwefligen Säure zu Schwefelsäure gebildeten Stickoxyde werden indeß $\mathrm{im}$ Verlauf des Kammerprozesses und im Gay-Lussac in gleicher Weise wieder nutzbar gemacht, bedeuten als solche also keine Verluste. Die für die Oxydation der $\mathrm{As}_{2} \mathrm{O}_{3}$ notwendigerweise ebenso einzusetzenden Salpetersäureverluste wie für die Oxydation der $\mathrm{SO}_{2}$, sind bei dem kolossalen Überschuß von $\mathrm{SO}_{2}$ über $\mathrm{As}_{2} \mathrm{O}_{3}$ aber so überaus minimale, daß sie mit Recht zu vernachlässigen sind, es sei denn, $\mathrm{N}$ i e d e $\mathrm{n} f \ddot{u} h_{\mathrm{r}} \mathrm{r}$ lege auch in dieser Beziehung Wert auf homöopathische Dosen. - Eine weit erheblichere Oxydationswirkung auf arsenige Säure als Glover und Kammer äußert der von schwefliger Säure nahezu freie Gay-Lussac. Ist die Annahme berechtigt, daB bei diesem Vorgang relativ viel nicht oxydier- und kondensierbares Stickoxydul entsteht, so würde sie einen weiteren Grund zur Ablehnung des $\mathrm{N}$ i ed e $\mathrm{n} \mathbf{f} \ddot{\mathrm{u}} \mathrm{h} \mathrm{r}$ schen Systems bedeuten müssen, das wegen derfortlaufenden Wiederbenutzung der Berieselungssäure im GayLussac immer größere Mengen arseniger Säure zur Oxydation bringt.

Hinsichtlich der Platzfrage des Ventilators habe ich meine Ansicht, die der $\mathrm{N}$ i e d e $\mathbf{n}$ f ü h r schen im allgemeinen entspricht, bereits geäußert; auch darin stimme ich ihm bei, daß ein gewisser Überdruck zur Úberwindung stagnierender Gasmassen, zur besseren Mischung der Gase usw. in bezug auf die Produktion nicht ohne Einfluß sein könne; nur glaube ich nicht, daß einem Mehr von 3-4 mm jene Leistungen zuzuschreiben seien, wie Niedenf $\ddot{\text { i }} \mathrm{h} \mathbf{r}$ behauptet, und die er lediglich für sich in Anspruch nimmt, und entschieden verwahren muß ich mich nochmals gegen die Zumutung, Fachleute glauben machen zu wollen, die durch einen Mehrdruck von 3-4 mm erzielte höhere Reaktionstätigkeit könne eine auch nur nachweisbare Wirkung haben. Das in diesem Punkte jetzt schon erheblich weniger sichere Auftreten $\mathrm{N}$ i ed e $\mathrm{n} f$ ii h r s legt übrigens die Vermutung nahe, er halte wenigstens in diesser Beziehung, den Rückzug für geboten.

Daß bei einer Höhe von $2300 \mathrm{~m}$ über dem Meeresspiegel, also bei nur $577 \mathrm{~mm}$ Normalquecksilberdruck, d. h. bei $183 \mathrm{~mm}$ Quecksilbersäule oder bei ca. $2500 \mathrm{~mm}$ Wassersäule unter der Norm, die Raumleistung der Kammern eine erheblich geringere sein muß als auf Meereshöhe, dürfte nicht zu bestreiten sein. Gerade aber aus dem Umstande, daß bei einer derartigen Höhe die Fabrikation immer noch lohnend gewesen, daß die Produktion nicht auf Bruchteile eines $\mathrm{kg}$ gesunken -.. uud das müßte der Fall sein, wären $\mathrm{L} \ddot{\mathrm{u}} \mathrm{t}$ y s und $\mathrm{N}$ i e d e $\mathrm{n} f \ddot{u} \mathrm{~h} \mathrm{r}$ s Behauptungen richtig - - wird es ersichtlich, welcher Wert ihnen eben beizumessen ist. Bei dem $\mathrm{N}$ i e d e $\mathrm{n} f \ddot{\mathrm{u}} \mathrm{h} \mathrm{r}$ schen PIus an U̇berdruck von $4 \mathrm{~mm}$ Wassersäule müßte cin um etwa $3,4 \mathrm{~m}$ tieferes 
Niveau genügen, um ohne diesen künstlichen Überdruck in dieser Beziehung dieselben Resultate zu erzielen. Sollte es wirklich notwendig sein, noch ausführlicher-hierauf einzugehen, um $\mathrm{N}$ i ed en $\mathrm{f} \ddot{\mathrm{u}} \mathrm{h} \mathrm{r}$ zu überzeugen, daß er sich in diesem für sein patentiertes Verfahren wesentlichen Punkte einer großen Selbsttäuschung hingegeben! Meinen Einwand, jedes Arbeiten mit außergewöhnlichem Überdruck habe nach einiger Zeit des Betriebes, sobald die geringsten Risse und Sprünge sich in den Kammern zeigen, von selbst aufzuhören, hat er vorgezogen mit Stillschweigen zu übergehen. In der Annahme, er akzeptiert diesen meinen Einwurf als unwiderlegbar, kann ich es mir nicht versagen, in bezug auf letzte beiden Fakta ihn an $\mathbf{H}$ or a z's schönen Spruch zu erinnern : ,Parturiunt montes, nascetur ridiculus mus".

Wie fast alle Angriffe $\mathrm{N}$ i ede $\mathrm{n} f \mathrm{u}$ h r s auf flüchtiger, resp. unverstandener Lektüre meiner Abhandlung beruhen, so auch der, ich hättè behauptet, der aus dem ersten Turm frei werdende Wasserdampf müsse im 2. Turm sämtlich kondensiert werden. Ich habe vielmehr gesagt: ,Es liegt Gefahr vor, - unter der Voraussetzung, daß sämtliche Kammersäure zur Konzentration gelangt, was nach $J$, ü t y s Ausführungen klugerweise nicht beabsichtigt zu sein scheint -, daß die aus dem Konzentrationsglover in den Denitrierglover übertretenden Wasserdämpfe.....mehr oder weniger zum Niederschlag kommen und die. unten abfließende Säure mehr verdünnen werden, als der Konzentrationsglover nachher zu verdampfen vermag". L ü t y basierte seine Berechnungen auf die Fabrikation von $60^{\circ}$ iger Säure. Meine Forderung ist demnach berechtigt, daß $\mathrm{N}$ i ed e $\mathrm{n} f \ddot{\mathrm{u}} \mathrm{h} \mathrm{r}$ nachweise, wie er imstande ist ohne besondere Kosten dieser Behauptung zu entsprechen. Aus seinen gewundenen Erklärungen: ,Größere Mengen Kammersäure im l. Apparat", ,etwas Kammersäure im 2. Apparat", geht in keiner Weise hervor, er gedenke dieser Anforderung ohne weiteres gerecht zu werden. Schaltet er etwa Konzentrationspfannen ein, so wäre $L$ ü $t$ y der Vorwurf zu machen, daß er diese besonderen Konzentrationskosten vernachlässigt habe, was um so weniger verzeihlich wäre, als er seine Berechnungen zu Vergleichen benutzte.

Schon aus dem Zugeständnis, praktischerweise es aufgegeben $\mathrm{zu}$ haben, im 2. Glover eine vollkommene Denitrierung zu erzielen (nach L ï t y zieht man die Säure mit $0,2 \%$ (?) $36^{\circ}$ iger $\mathrm{HNO}_{3}$ ab), geht hervor, daß man im anderen Falle, wenn völlige Denitrierung erzwungen wird, derart dünne Säuren erhält; daß sie im 1 . Glover nicht mehr zu konzentrieren sind. Wo bleibt d a n $\mathrm{d}$ i e Konzentrationder Hauptmengeder $\mathrm{K}$ a $\mathbf{m} \mathrm{m}$ ers ä u re?

Meine damals rein theoretischen Bedenken fanden bereits eine Stützo durch Dr. Schli e b s' Mitteilungen aus der Praxis, die zwei Fälle betrafen, in denen der Konzentrator ungenügende Verdampfungsleistung aufwies. Auf $\mathrm{N}$ i eden$\mathrm{f}$ ï $\mathrm{h} \mathrm{r}$ s Widerlegungen zu antworten, muß ich natürlich Dr. S c h 1 i e bs überlassen; ich bin jedoch in der Lage, zwei analoge Fälle hinzuzufügen, die weitere interessante Illustrationen zu diesem Punkto bieten.

Der erste betrifft die sowohl von Lü t y als besonders jetzt wieder von $\mathrm{N}$ i ed en $\mathrm{f} u ̈ \mathrm{~h} \mathrm{r}$ angeführte Anlage in Roubaix (Wattrelos), in der es nach ihnen gelungen ist, dic glänzenden Resultate zu erzielen, auf die $\mathrm{N}$ i e d e $\mathrm{n} f$ ü $\mathrm{hr}$ sich in erster Linie als Referenz beruft. Es wird mir als ein Faktum mitgeteilt, bis nicht allzulange vor der Zeit des L ï t y schen Vortrages seien trotz. der hohen Eintrittstemperatur von $400^{\circ}$ und mehr der Gase in den 1. Glover alle Bemühungen vergeblich gewesen, durch Konzentration in ihm eine genügend starke Gay-Lussacsäure zu erzielen, so daß diese durch Zusatz stärkerer Säure aus anderen Systemen gewonnen werden mußte. Erst durch Zwisehensehaltung von großen Gasfiltern zwischen die Glover, die gleichzeitig als Kondensatoren dienten, und aus denen dauernd eine Säure von ca. $40^{\circ}$ Bé. ablief, - ebenso wie aus dem Ventilator, wie $\mathrm{N}$ i e $\mathbf{d}$ e $\mathbf{n} \mathbf{f}$ ü $\mathbf{h} \mathrm{r}$ angibt war es möglich, im 1. Turm eine Säure von $60^{\circ}$ Bé. zu erhalten. $-\mathrm{N}$ i ed e $\mathrm{n} \mathrm{f} \ddot{\mathrm{u}} \mathrm{hr}$ wird billigerweise wohl zugeben müssen, meine damalige $\mathrm{Be}$ hauptung, -- die unter der Voraussetzung abgegeben war, sämtliche Kammersäure gelange in die Glover zur Konzentration, - daß ein Teil des Wasserdampfes im 2. Glover sich kondensieren müsse, bedarf nicht einmal jener vorsichtigen Einschränkung, um dennoch berechtigt zu sein. - Hätte jene Säure von $40^{\circ}$ Bé. nicht in der Düngerfabrikation Verwendung finden können, so hätte man sie verloren geben müssen. An eine völlige Konzentration der Kammersäure war unter diesen Umständen natürlich nicht im entferntesten zu denken, was dort, in einer Düngerfabrik, auch nicht verlangt wurde. $O b$ und in wieweit es später gelungen, die Konzentration der GayLussacsäure auf eine rationellere Weise zu ermöglichen als durch das primitive Mittel von Kondensation zwischen den Glovern, wodurch die Gase auf eine Temperatur von $30-40^{\circ}$ gebracht werden, dic neben anderen Mängeln eine auch nur annähernd völiige Denitrierung der Nitrose im 2. Glover ausschließen muß, entzieht sich meiner Kenntnis.

Eine zweite Anlage, auf dio die in Rede stehenden Nachteile und Unzuträglichkeiten teilweise noch heute zutreffen, ist im März vergangenen Jahres in Betrieb gekommen. Ich vermute, daB es dieselbe Anlage ist, von der L $\ddot{u}$ t y auf S. 1260-1261 seines Vortrages sagt: ,Von besonderem Interesse sind die Resultate des neuesten, nach dem $\mathrm{N}$ i e d e $\mathbf{n}$ $\mathrm{f} \ddot{\mathrm{u}} \mathrm{h} \mathrm{r}$ schen Verfahren in Betrieb genommenen Schwefelsäuresystems, das in demselben Gesamtarrangement und Dimensionen, wie das mit Fig. 3 illustrierte, ausgeführt wurde und sich von letzterem nur dadurch unterscheidet, daß bei ihm dic Gase in die runde Kammer nicht tangential, sondern von der Decke aus eingefïhrt wurden. B e i d e A n lagen zeigen das völlig gleiche Bild des Arbeitsverlates und der Produktionsleistungenc.

Daß diese Behauptung nichts weniger als den realen Verhältnissen entsprechen dürfte - es sei denn, Anlago nach Fig. 3 lasse gleichfalls orhoblich zu wünschen übrig - wird später gezeigt werden. Obwohl die Ofengase hicr mit $450^{\circ}$ in den 1. Glover treten und ihn mit ca. $80^{\circ}$ verlassen, gelang es in ihm, wie man mir mitteilt, nicht, die aus dem 
2. Glover ausfließende denitrierte Säure von 54 bis $55^{\circ}$ Bé. auf die Gay-Lussacstärke zu bringen, so daß auch hier Säure aus anderen Systemen zu Hilfe genommen werden mußte unter Verwendung eines großen Teils der auf nur ca. 56 ${ }^{\circ}$ Bé, konz. alten Gay-Lussacsäure zu anderen Zwecken. Ich bin gern bereit, falls $\mathrm{Ni}$ edenführ im Zweifel sein sollte, um welche Anlage es sich handelt, ihm diese namhaft zu machen, zumal jene Mitteilungen nicht allein zu L $\mathrm{L}$ t ys obigen Behauptungen im Gegensatz zu stehen scheinen, sondern auch mit den jüngsten Versicherungen $\mathrm{N}$ i ed e $\mathrm{n} f \ddot{u} \mathrm{~h} r \mathrm{~s}$ : ,Heute kenne ich in dieser Beziehung keine Schwierigkeiten, vielmehr habe ich bei einem System in Osterreich-Schlesien usw. " ohne weiteres nicht. in Einklang zu bringen sind.

Über $55^{1} / 2^{\circ}$ Bé. wird die aus dem 2. Glover abfließende Säure sofort nitros, bei $57-58^{\circ}$ Bé. enthält sie in 1 Liter bis zu $12 \mathrm{~g} \mathrm{HNO}_{3}$ (im Durchsehnitt $0,4-0,8 \%$ von $36^{\circ}$, nicht etwa $0,2 \%$ wie $L$ ü t y angab), so daß es nicht angängig ist, soll völlige Denitrier ung erzielt werden, sie durch $\mathrm{Zu}$ satz von weniger dïnner Säure stärker zu erhalten als $55^{\circ}$ Bé. Grund ist of $f$ en bardie $\mathrm{f} \ddot{\mathrm{u}} \mathrm{r}$ $S t a ̈ r k e n$ von über $55^{\circ}$ Bé. zu niedrige Eintrittstemperatur der Gasevon n u $r \quad 70-80^{\circ}$. In Übereinstimmung hiermit, als auch mit meiner früheren Annahme, geben $L \ddot{u}$ t $y$ und $\mathrm{N}$ i e d e n fü h r an (diese Z. 15, 251 [1902]), die denitrierte Säure sei so zu halten, daß sie nur $54^{\circ}$ Bé. stark würde. Außerdem sagen sie auch, $\mathrm{da} B$ von dieser denitrierten Säure nur ein Teil zur Berieselung des 1. Glovers zu verwenden sei, wo sie die Gase reinige und selber konzentriert werde.

Der Glover ist aber nicht nur ein Apparat zur Denitrierung der $\mathrm{Nitrose,} \mathrm{sondern} \mathrm{ersoll} \mathrm{auch} \mathrm{ein}$ ganzhervorragender Schwefelsäurebildner sein, und dieser Voraussetzung scheint mir trotz der $\mathrm{SO}_{2^{-}}$ reichen Gase bei einer Gaseintritts. temperatur von nur $70-80^{\circ} \mathrm{nicht}$ entsprochen zu sein.

Auf diesen speziellen Punkt ausführlicher einzugehen und ihn theoretisch $\mathrm{zu}$ begründen, würde für heute zu weit führen; ich muß mir dieses Thema für einen späteren Artikel vorbehalten. Heute sei nur daran erinnert, daß der um so viel fruchtbarere Schwefelsäurebildungsproze $B$ im Glover in ganz anderem Sinne und auf Grund anderer Vorausetzungen hinsichtlich der Temperatur usw. verläuft als der Kammerprozeß. Wenn aber, wie durch obige Fakta erwiesen, für gewisse uns hier interessierende Säurestärken die unterste Denitrierungsgrenze bei einer Temperatur von $70-80^{\circ}$ der Eintrittsgase erreicht wird, muß eben das Denitrierungs- oder Reduktionsoptimum für diese Säurestärken höher liegen, von dessen möglichst Erreichen resp. Ausnützen in Verbindung mit dem sich möglichst Nähern an das Nitrosylschwefelsäurebildungs- oder Oxydationsoptimum ja die Kapazität des Glovers als Schwefelsäurebildners abhängt.

Wird die Wirkung des Glovers als Schwefelsäurebildner herabgesetzt, - das event. Faktum, daß die Gesamtproduktion des Systems gestiegen, kann dieses Argument nicht beeinflussen -, so wird es verständlich, daß seine scheinbare Konzentrationswirkung, die in erster Linie auf Aufnahme von im Glover gebildeter Schwefelsäure ${ }^{4}$ ) beruht, entsprechend vermindert werden muB. $\mathrm{N}$ i e d e $\mathbf{n}$ f ü h $\mathbf{r}$ gibt übrigens selber an, bei Mitverdampfung größerer Mengen Kammersäure (?!) im 1. Glover, also bei Aufgabe dort etwa einer Säure von $56-58^{\circ}$ Bé., würde im 2. Glover die Stärke der allein aufgegebenen Nitrose von $60-61^{\circ}$ Bé. auf 58 bis $59^{\circ}$ Bé. sinken. Das heiBt nichts anderes, als da $B$ in Berücksichtigung der bei einer Temperatur von $70-80^{\circ}$ warmen Oxydationszone in die Kammer fortgeführten beträchtlichen Wasserdampfmengen, die Aufnahme durch gebildete Schwefelsäure nur eine ganz minimale ist im Gegensatz zu den großen Mengen Säure, die ein normaler Glover bildet. Da aber andererseits bei $58-59^{\circ}$ Bé. und jener niedrigen Reduktionstemperatur von $70-80^{\circ}$ völlige Denitrierung nicht im entferntesten erzielt wird, wird im 2. Glover noch etwas (!?) Kammersäure zugegeben, so daß unten eine Säure von $57^{\circ}$ Bé. resultiert, die, wie wir wissen, immer nooh beträchtliche Salpetersäuremengen enthält. Diese kommt nun im 1. Glover zur Konzentration, und zwar nach $\mathrm{Ni}$ ed enführ zusammen mit einer größeren Menge (?!) Kammersäure, so daß die Berieselungssäure hier etwa $56-56,5^{\circ}$ Bé. (?) stark sein mag.

$\mathrm{L}$ u $\mathrm{n}$ g hat bereits darauf hingewiesen, daß die Konzentrationswirkung des Glovers durch Wasserverdampfung überschätzt worden ist. Die Erfahrung in Roubaix und in der von mir zitierten Anlage bestätigen seine Ansicht. Gelingt $\mathrm{N}$ i e d e $\mathrm{n}$ -

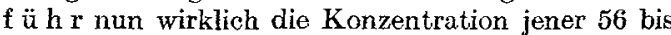
$56,5(?)^{\circ}$ Bé.-Säure im 1. Glover, so ist es klar, daß sie besonders der bekannten Schwefelsäurebildung nach der Glovergleichung und dem Gloverprinzip $\mathrm{zu}$ verdanken ist, die bei Gegenwart der im 2 . Glover nicht völlig denitrierten Nitrose, ihrem Gehalte von $0,4-0,8 \% \mathrm{HNO}_{3}$ und der Temperatur der heißen Eintrittsgase entsprechend, durch Anreicherung und Wasserverdampfung in alter Weise wirksam ist. Denn daß ein normaler Glover, der durch mehr oder weniger energische Reaktionstätigkeit entsprechende Wärmemengen produziert, bei derselben Gaseintrittstemperatur auch hinsichtlich der Wasserverdampfung crheblich leistungsfähiger sein muß, als ein lediglich im Sinne der Wasserverdampfung tätiger Turm, dürfte selbstverständlich sein.-Abgesehen davon, daß ein gewisser Teil der Salpetersäure den Versicherungen entgegen somit nicht den hohen Temperaturen und der Berührung mit Flugstaub entzogen wird, so arbeitet $\mathrm{N}$ i c d en $\mathrm{f} \mathrm{ü} \mathrm{hr}$ auch außerhalb des Rahmens seines eigenen Verfahrens, das laut Patentanspruch sich auf einen Konzentrator und einen Denitrifikator beschränkt. Hier sind aber ein völliger Glover und ein nachgestellter Vordenitrifikator vorhanden! Der Nachteil der Unmöglichkeit der Konzentration der gesamten Kammersäure wird aber verbleiben, und ich möchte behaupten, der Einfluß der unvorteil-

4) L u n g e s Ansicht (I., 592 [1903]), auch Anhydrid würde hier absorbiert, dürfte auf Grund der mit der Absorption des Kontaktanhydrids gemachten Erfahrungen eine Berichtigung wohl insofern angebracht erscheinen lassen, als es sich hier um nur Absorption des allergeringsten Teils des Anhydrids handeln kann. 
haften Arbeitsbedingungen wird trotzdem so groß sein, daß die Menge der konz. Kammersäure nur geringfügig sein kann. Ein weiterer großer Nachteil dieses Arbeitsganges ist dann aber dadurch gegeben, daß der Gay-Lussacturm mit fast immer derselben Säure gespeist wird, die sich im 1. Glover an Eisensalzen, an Arsen usw. immer mehr anreichert, so daß nach einer gewissen Betriebszeit ihr Ersatz durch Säure aus anderen Systemen oder ihre umständliche Reinigung erforderlich wird.

Vorteilhafter wïrdesich meines Erachtens der Gang offenbar ge stalten, wenn auf irgend eine Weise die Temperatur der Eintritsgase in den 2. Glover so hoch gehalten werdenkönnte, daBsie etwa 130 bis $150^{\circ}$ oder mehr betrüge; bei diesen Temperaturen w ürde der Gloverschwefelsäurebildungsprozeß in alter Weise einsetzen, und voraussichtlich würde sich so eine Anreicherung gröberer Mengen $\mathrm{K}$ a m m ersäure ermöglichen lassen. Auf Grund dieser Ausführungen glaube ich ein Recht zu haben zu der Behauptung, Ni ed e n $\mathrm{f} \ddot{\mathrm{u}} \mathrm{h} \mathrm{r} \mathrm{s}$ Gloverteilung ist ein Unding, denn sein Denitrifikator denitriert unvollkommen, und sein Konzentrator leistet nicht im entferntesten das, was von einem normalen Glover in dieser Beziehung erwartet werden muß.

$\mathrm{N}$ i e d en fü hrs Auseinandersetzungen über Bleiabnutzung beim Arbeiten mit Intensivsystemen, die er mit apodiktischer Sicherheit „,als Widerlegungen" bezeichnet, kann ich als solche nichts weniger als anerkennen. Abgesehen davon, daß seine Ansichten auch zu denen $\mathrm{L} u \mathrm{n}$ ges, wie gez eig $t$, im Gegensatz stehen, vermag ich nicht einzusehen, warum der allgemein gültige Satz: ,,Jeder Gegenstand erfährt durch größere Inanspruchnahme auch schnellere Abnützung", in bezug auf die Abnützung der Bleikammern nicht zutreffen soll.

Die Erkenntnis unserer Zeit geht immer mehr dahin, daß selbst „das Leben der Metalle“ ein beschränktes sei, und daß ihr molekularer Zusammenhang desto schneller gelockert wird, je mehr sie Erschütterungen, Stößen usw. ausgesetzt sind, und je stärker und schneller diese erfolgen. Spröde Metalle unterliegen diesen Einflüssen natürlich erheblich schneller als nachgiebige und weiche. Es ist aber nur folgerichtig anzunehmen, $\mathrm{da} B$ auch das Blei ein entsprechendes Verhalten zeigen wird, $\mathbf{d} . \mathbf{h}$. daB es durch die bei stärkerem Drucke notwendigerweise auch stärkeren Vibrationen schneller in seinem molekularen Zusammenhang gelockert wird, so daß, wenn es durch diese Einflüsse auch keine direkte Tension zum Platzen oder Reißen zeigen mag, es doch den Bruch- und Reißbestrebungen und den chemischen Einflüssen auf die Dauer geringeren Widerstand wird entgegensetzen können. Ohne behaupten zu wollen, gerade dieser Umstand sei für jene Anlage in Roubaix zutreffend, so wird es mir doch als Faktum bezeichnet, daß schon nach kurzer Betriebszeit die Kammern dort ungewöhnlich stark mitgenommen waren, die umfassende Reparaturen benötigten, wie das auch E. H a r t- $m$ a $n n$ und $F$. B e $n k$ e $r$ in ihrem jüngsten Aufsatze bestätigen (diese $Z .19,133$ [1906].).

Abgesehen davon, daß durch Ventilatorenbetrieb der Kammergang zweifelsohne ein geregelterer und gleichmäßigerer wird, muß doch entschieden in Abrede gestellt werden, als ob $\mathrm{N}$ i ede $\mathbf{n}$ fü hrs Behauptungen berechtigt seien, daß ohne Ventilatorbetrieb unaufmerksame Bedienung, schroffe Varianten in der Salpetersäure- und Dampfzufuhr usw. an der Tagesordnung und Anläße zu frühzeitiger Bleiabnutzung gewesen. Was bedeuten diese in Beziehung auf Bleiabnutzung erzielten kleinen Vorteile gegen die oben und im folgenden angeführten großen Nachteile!

$\mathrm{N}$ i e d e $\mathbf{n} \mathbf{f}$ ü h $\mathbf{r}$ s Behauptung, , bei träger Reaktion, kleiner Raumleistung ${ }^{\natural t}$ und ,niedriger Gastemperatur" werde das Kammermaterial eher korrodiert, als beim Gegenteil, ist in dieser Form so haltlos, daß ich wirklich nicht verstehe, wie ein Fachmann sie aufstellen kann. Was er über die Möglichkeit der Salpetersäurebildung bei niedrigen Temperaturen sagt, traf wohl früher für die letzte Kammer zu, hat aber seit Einführung des Gay-Lussacturmes, besonders jetzt im Zeitalter der Intensivproduktion, selbst für diese keine Berechtigung mehr ( $\mathrm{L}$ u $\mathrm{n}$ g e I., 611 [1903]). An welcher Stelle gibt es denn in einem System (wenn nicht uralter Konstruktion) mit normalem Gange so niedrige Temperaturen, die niedriger sind, als die auch nach seinem Arbeitsgange sich ergebenden, oder die in dieser Hinsicht besonders zu fürchten wären? Gerade hohe Temperaturen, kräftige Reaktion, starke Raumausnutzung bedingen den Verschleiß des Bleimaterials, wie aus dem Verhalten der 1. Kammer hervorgeht. Um wieviel schneller müssen diese Faktoren sich noch bei dem $\mathrm{N}$ i e d e $\mathrm{n} f$ ï $\mathrm{h} \mathrm{r}$ schen System geltend machen, bei dem ein wesentlicher Teil der im Glover nicht mehr in alter Weise stattfindenden Schwefelsäurebildung in die Kammern verlegt wird und diese weit über die bisher zulässige Grenze hinaus überlastet, bei dem nicht nur die Vibrationen durch höheren Druck die schützenden Bleisalzüberzüge zu zerreißen und neue Metallflächen bloßzulegen vermögen, sondern deren Wirkung auch geeignet ist, die molekulare Konstitution des Bleies zu lockern, so daß es chemischen Angriffen leichter zugänglich wird.

Werden diese Deduktionen als berechtigt anerkannt, so erübrigt sich die Frage der ,ganz beträchtlich niedrigeren Reparaturkosten ". Was tuts, wenn das Bleiquantum nur die Hälfte beträgt, und wenn die Notwendigkeit der Reparaturen so groß, und diese so umfassend sind, wie angenommen werden muß, und wie der Fall in Roubaix zu bestätigen scheint! Volle Veranlassung dürfte somit gegeben sein - ebenso wie man in $L \ddot{u}$ t y s Selbstkostenpreisaufstellung (diese Z. 18, 1262 [1905]) die Amortisationsquote für $100 \mathrm{~kg} \mathrm{H}_{2} \mathrm{SO}_{4}$ nicht auf 0,2672 , sondern auf $0,4-0,5 \mathrm{M}$ festzusetzen habe - , die Reparaturkosten nicht mit 0,0667 , sondern mindestens mit 0,12 einzuschätzen.

Dabei darf nicht vergessen werden, daß auf Grund jener Aufstellung einer Produktion von $80000 \mathrm{~kg}$ 50iger Säure ein Vergleich überhaupt un. zulässig ist, weil derartige Riesensysteme, wenn sie gebaut sind oder wenn sie gebaut werden sollten, nicht die Regel, sondern die allergrößte Ausnahme 
wären. Hinsichtlich der Genauigkeit, mit der diese Aufstellung gemacht, sei übrigens noch darauf hingewiesen, daß von laufonden Lizenzgebühren resp. von der Amortisation eines einmaligen Lizenzhonorars mit keinem Worte oder Pfennig die Rede ist. Es ist doch nicht gut anzunehmen, $\mathrm{N}$ i c d en f ü h r s Ansprüche für Erbauung einer solchen Anlage seien so klein, daß sie mit Recht zu vernachlässigen sind.

Nachdem ich so keine einzige der gegen meine Einwände gerichteten Ausführungen $\mathrm{N}$ i e d e n f ü h r s unberücksichtigt gelassen, glaube ich mit Recht behaupten zu dürfen, daß ich vollste Veranlassung habe, meine bisherigen Einwände in allen Punkten durchaus aufrecht zu erhalten.

Ferner sei noch darauf hingewiesen, wie $I$ ü $t$ y in seinem bekannten Vortrag wörtlich sagt: ,Ein weiterer Vorzug des Verfahrens ist, daß es die $\mathrm{Zusammenkombinierung} \mathrm{versehie-}$ denerheterogener Ofensysteme und ebenso die gleichzoitige Verarbeitung vers ch i e. dener Schwefelrohstoffeanstands lo s zulälst. So hat $\mathrm{N}$ i e de $\mathrm{n} f$ ü h $\mathrm{r}$ in Roubaix (Wattrelos) 3 Ofensysteme..... o hn e da $B$ sich hieraus Betriebsschwierig $k$ eiten ergaben......! Weiterhin wird behauptet: ,Vorher möge noch auf die Wichtigkeit des Verfahrens des D. R. P. 140825 auch für alle bestehenden Anlagen verwiesen werden, daß nämlich dasselbe nicht nur erlaubt, schon existierende Systeme jeglicher Ausführungsweise mit minimalen Unkosten lukrativer zu gestalten, sondern auch die feblerhaften Anlagen in Ordnung zu bringen".

Über die Anlage in Roubaix habe ich bereits berichtet, daß nach mir gewordenen Mitteilungen bis nicht unlange vor $L$ ü t y s Vortrag die Schwierigkeiten bzgl. der Konzentration der Gay-Lussacsäure und erst recht der Kammersäure nicht gelöst waren. Ob das ursprünglich von $\mathrm{B}$ en ker errichtete System wirklich eine so überaus geringe Leistung aufgewiesen, wie $\mathrm{N}$ i e d e $\mathrm{n} f \ddot{\mathrm{u}} \mathrm{h} \mathbf{r}$ behauptet, so da $B$ sein Eingreifen aus diesem Grunde erforderlich geworden, lasse ich, als nicht in der Lage, diese Behauptungen zu kontrollieren, dahingestellt. Ist, wie anzunehmen, das nicht der Fall, so dürfte die Berichtigung von beteiligter Seite wohl nicht lange auf sich warten lassen. Immerhin scheinen die in dieser Anlage damals erzielten Resultate - es wird mir auch berichtet, die Betriebsschwierigkeiten seien außerordentliche gewesen, und der Salpetersäureverbrauch habe $1,0-1,2 \% 36^{\circ}$ iger Salpetersäure betragen -, nicht derart gewesen zu sein, daß sie $\mathrm{L}$ ü t y zu jenen so empfehlenden Anpreisungen berechtigten. Auch am Schlusse seines gegenständlichen Artikels gibt $\mathrm{Ni}$ e d e $\mathrm{n} f \mathrm{u} \mathrm{h} \mathrm{r}$ an, daß er in nächster Zeit wieder Gelegenheit haben werde, über Betriebsresultate mit Anlagen für verschiedene Rohstoffe, die unter teilweisen recht heterogenen allgemeinen Verhältnissen arbeiten, zu berichten. Ich weiß dahingegen bestimmt, eine solche Anlage ist bereits im Laufe des vergangenen Sommers in Betrieb gekommen, und möchte demnach meiner Verwunderung Ausdruck geben, daß $\mathrm{N}$ i ed e $\mathrm{n} \mathrm{f} u ̈ \mathrm{~h} \mathbf{r}$ mit der Publizierung der für derartige Anlagen erzielten Resultate auf die Zukunft vertröstet, während ihm doch Gelegenheit gegeben ist, den Beweis seiner Behauptungen schon sofort anzutreten.
Ebenso wie für die Anlage in Roubaix

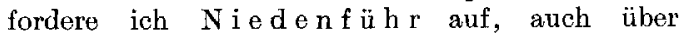
die zweite von L üty erwähnte Anlage, die in Petrowitz (Österreich-Schlesien), nähere Mitteilungen zu machen, und wenn angängig, durch zeitlich getrennte Monatsresultate. Die Produktion soll hier $11-12 \mathrm{~kg}$ betragen; interessant wäre es aber, zu hören, mit welchen Mitteln sie erreicht wird, ob Plattentürme vorhanden, und welches das Verhältnis ibres Kubikinhaltes zum Kammerraum ist, wie hoch die Kammern sind, wieviel Salpetersäure bei jener Produktion im Umlauf und welches ihr Verlust ist, wieviel Kammersäure zur Konzentration gelangt, und mit wieviel Gehalt an Salpetersäure, von $36^{\circ}$ Bé. die aus dem 2. Glover ablaufende Säure von $x^{\circ}$ Bé. in den 1. Glover gelangt. Durch eine Reibe einfacher Versuche wird sich hier anch mit Leichtigkeit feststellen lassen, wolchen Einfluß der höhere Überdruck auf die Produktion ausübt.

Daß derartig hohe Intensiv produktionen keineswegs lediglich Eigentümlichkeit des $\mathrm{N}$ i ed e $\mathbf{n}$ $\mathrm{f}$ ü $\mathrm{h} \mathbf{r}$ schen Systems sind, - ein Faktum, auf das Dr. S c hlie bs bereits aufmerksam gemacht, geht aus $\mathbf{F a l d i n g ~ s}{ }^{5}$ ) Mitteilıng (Min. Ind., 602 [1901]) hervor, der berichtet, das Tangentialsystem in Baltimore habe ohne Ventilator, ohne Wasserzerstäubung und ohne Reaktionstürme cine Produktion von $6,2 \mathrm{~kg} \mathrm{H} \mathrm{SO}_{4}$ gleich $10 \mathrm{~kg} 50^{\circ}$ iger Säure geleistet. Mit jenen Hilfsmitteln müßte die Produktion, falls das empfehlenswert erschienen wäre, zweifelsohne mit Leichtigkeit auf $12-13 \mathrm{~kg}$ zu steigern gewesen sein.

Auch über die dritte von $L$ ü t y besprochene Tangentialanlage (Fig. 3), mit der Dr. S c h li e bs zu arbeiten Gelegenheit hatte, wären detaillierte Angaben erwïnscht. Aus der durch $\mathrm{N}$ i e d e $\mathrm{n} f$ ï $\mathrm{h} \mathrm{r}$ publizierten Meinungsäußerung des Nachfolgers von Dr. S chlie bs geht durchaus nicht hervor, daß dieser jenem außergewöhnlichem Überdruck von 3-4 mm Wassersäule einen ganz besonderen Finfluß auf die Produktion zuspricht.

Was im übrigen von $\mathrm{N}$ i eden $\mathrm{f}$ ü h $\mathrm{r}$ s Behauptungen und Versicherungen zu lialten ist, möge folgende Gegenüberstellung zweier sciner Mitteilungen erweisen. Wir erfahren von ihm, jetzt im Jahre 1906, auf Grund seines persönlichen Eingreifens in die Leitung jenes letzten Systems, nach Dr. Schliebs Fortgang, sei die Produktion sofort eine viel bessere geworden, und $9,5 \mathrm{~kg} 50^{\circ}$ iger Säure pro cbm Kammern- und Plattenturm raum seien erreicht worden, Leistungen, die er als solche bezeich-

5) Von Interesse wird es sein zu hören, was $\mathrm{F}$ a $1 \mathrm{~d}$ i $\mathrm{ng}$, dessen Sachkenntnis und Erfahrungen $\mathrm{N}$ i e d e $\mathbf{n} \mathrm{f} \ddot{\mathrm{u}} \mathrm{h} \mathbf{r}$ anerkennt, in einem Schreiben an mich über Arbeiten mit Ventilatoren sagt : „I us e the fans entirely and simply for maintaining a uniform and com pletecontrol of the draftorspeed of the gas through the whole system. It is my experiencethat if the fans are used to materially increase the pressure in the eham. bers, that the results of such are dis a s trou s. " Dieses Urteil, dem alle mir bekannten maßgebenden Persönlichkeiton aus der Schwefelsäureindustrie bedingungslos zustimmen, bedeutet von autoritativer Seite eine glatte $A b$ lehnung des $\mathrm{N}$ i e d e $\mathrm{n} f \ddot{u}$ h $\mathrm{r}$ sehen Systems. 
net, wie siekeineder bisher erbauten Tangentialkammern a u $\mathrm{z}$ uweisen $\mathrm{g} e \mathrm{~h} a \mathrm{~b}$ t $\mathrm{h}$ a be. Im krassen Gegensatz zu diesen Behauptungen stehen indes seine eigenen Angaben in $\mathrm{Nr} .1$ der von ihm herausgegebenen „Mitteilungen aus der Praxis chemischer Industrien"vom Jahre 1903 : „Zur Charakterisierung der durch die Kombinierung von Bleikammern mit Plattentürmen heute erreichten Maximalleistungen führe ich an, $d$ a $B$ bei einer von mir erbauten Anlage mit Meyerschen Tangentialkam mern pro cbm Reaktions ra u m 9-10 kg $50^{\circ}$ iger Säurefabriziert w urden". Das ist nicht seine damalige Ansicht, die, eventuell als durch die Zeit überholt, heute zu widerrufen wäre, sondern das ist ein von ihm selbst konstatiertes Faktum, auf Grund dessen er gerade für jenes System Freunde geworben!! Dabei waren damals weder Ventilatoren im Betriebe, - natürlich gab es erst recht keinen außergewöhnlichen t'berdruck -, noch wurde Wasserzerstäubung verwandt, ein Hilfsmittel, über dessen Verwendung $\mathrm{N}$ i ed e $\mathbf{n} f$ ü $\mathbf{h} \mathbf{r}$ sich übrigens in jenen Mitteilungen recht reserviert, ja sogar skeptisch äußert. Wären jene Hilfsmittel in rationeller Weise schon in diesem System auch ohne den außergewöhnlichen Überdruck, ohne die Ventilatorstellung zwischen zwei Glovern, in Anwendung gekommen, so würde wie im Baltimorer Falle die Produktion von 9 bis $10 \mathrm{~kg}$ leicht auf $12-13 \mathrm{~kg}$ zu steigern gewesen sein, wie sie $\mathrm{Nieden} f \ddot{u} h \mathbf{r}$ später in seiner unter Fig. 3 beschriebenen Anlage tatsächlich erreicht haben will, die er aber nun als eine Folge der Einführung seines Verfahrens in Anspruch nimmt. Alle seine Deduktionen über die Wertlosigkeit des Tangentialprinzips zerfallen auf Grund dieses von ihm selbst mitgeteilten Faktums in ein Nichts.

Eine vierte Anlage, in der sein System zur Anwendung gekommen, ist die, von der Dr. Sc hli e bs gleichfalls nichts Rühmenswertes zu erzählen weiß. Auf $\mathbf{N}$ i e d e $\mathbf{n} f \ddot{\mathrm{u}} \mathrm{h} \mathbf{r} \mathrm{s}$ Widerlegung, die allerdings nur auf einen Punkt eingeht, zu antworten, ist aber Dr. S chli eb s' Sache.

Eine weitere Anlage ist die von mir bereits erwähnte, im März 1905 in Betrieb gekommene, von der ich vermute, daß sie mit der vierten, von L ü t y erwähnten, identisch sei. Man sehreibt mir, $\mathrm{daB}$ ihre Leistungen in keiner Weise befriedigen. Nicht nur der Gloverbetrieb sei ein ungenügender (die aus dem 2. Glover abfließende Säure hat bei ca. $57,5^{\circ}$ Bé. immer noch $0,4-0,8 \% 36^{\circ}$ iger Salpetersäure, und Konzentration der Kammersäure ist fast ausgeschlossen), der Salpetersäureverbrauch sei nicht der erhoffte (etwa $0,9-1 \% \mathrm{HNO}_{3}$ von $36^{\circ}$ Bé.), auch die Produktion bleibe weit unter der in Aussicht gestellten, d. h. sie betrage nur ca. $8 \mathrm{~kg}$ pro ebm Kammerraum ohne die Plattentürme, im Sommer noch weniger. - $\mathrm{N}$ i e d en $\mathbf{f} \ddot{u} \mathrm{hr}$ hat übrigens in diesem Falle die tangentiale Gaseinführung in die runden Kammern zu einer Deckeneinführung gemacht, eine Idee, die er, wenn auch nicht in dieser so doch in variierter Form nach Lüty (Vortrag diese Z. 18, 1260 [1905]) zum Gegenstand einer weiteren Patentanmeldung machen will, obwohl gerade die hier erzielten schlechteren Resultate (vgl. die von ihm selbst mitgeteilten Resultate mit M e y e r s Tangentialsystem vom Jahre 1903) ein weiterer Beweis für den Wert des Tangentialprinzips sind.

Habe ich in meiner Abhandlung Beweise für die von $\mathrm{L}$ ü t y mitgeteilten Resultate verlangt, so war ich, - ich wiederhole es -, nachdem Niedenführ durch $\mathrm{L}$ ü t y s Publikationen in einer wissensehaftlichen Zeitschrift sein Verfahren zur Diskussion gestellt, nachdem er die Angelegenheit vor das Forum der Offentlichkeit gebracht, dazu durchaus berechtigt. Auf $\mathrm{N}$ i e d e $\mathrm{n} f \ddot{\mathrm{h}} \mathrm{h}$ s jetzige Ausflucht, ,ernstlichen Interessenten" wolle er gern Beweise zugänglich machen, bin ich genötigt zu antworten, er hätte dann logischerweise auch seine Anpreisungen lediglich an jene,,ernsten Interessenten " richten, seine Publikationen auf den Inseratenteil chemischer Zeitschriften beschränken und die wissenschaftliche Offentlichkeit mit seinen Publikationen meiden sollen. In seinen Behauptungen aber wissenschaftlich sein zu wollen und in seinen Beweisen rein geschäftsmäßig, ist eine Inkonsequenz.

Daß bei der Fülle der Widersprüche alle Veranlassung vorliegt, die Fakten ernstlich zu prüfen, geht aus Dr. S c h li e bs und den mir gewordenen und hier veröffentlichten Mitteilungen hervor.

Abgesehen davon, daß ich mehr wie je überzeugt bin, daß alle derartig übermäßig hohen Intensivproduktionen in bezug auf die lange Haltbarkeit, auf die Solidität der Kammern unrationell sind, abgesehen davon, daß diese unrationelle Wirtschaft aus den angegebenen Gründen (höherer Druck und Verlegung eines großen Teils der Produktion des Glovers in die Kammern) beim $\mathrm{N}$ i e d e $\mathrm{n} f \ddot{\mathrm{u}} \mathrm{h} \mathrm{r}$ schen System sich zu einer Art Raubwirtschaft herausbilden wird, abgesehen ferner davon, daß jedes Arbeiten mit außergewöhnlichem Utberdruck mach wenigen Jahren Betriebszeit bei Entstehung der ersten Risse usw. von selbst unterbleiben muß, habe ich den Beweis erbracht, daß $\mathrm{N}$ i e d e $\mathrm{n} f \ddot{\mathrm{u}} \mathrm{h} \mathrm{r}$ überhaupt nicht mehr unter den Bedingungen seines patentierten Verfahrens fabriziert, daß er sich vielmehr notgedrungen meinen in meiner vorigen $\mathrm{Ab}$ handlung gemachten Vorschlägen nähert, die jedermann instandsetzen, in gleicher Weise zu arbeiten wie er. Dazu liegt aber nicht die mindeste Veranlassung vor! Im Gegenteil! Der einzige eventuelle Vorteil, den $\mathrm{N}$ i e d e $\mathrm{n} f \ddot{\mathrm{u}} \mathrm{h} \mathbf{r}$ erzielt, die geringe Ersparnis an Salpetersäure wird für die meisten Interessenten mehr als aufgewogen durch den erheblichen Nachteil, daß das System nur wenig $60^{\circ}$ ige Säure liefern kann, wozu noch die Beschwerlichkeit der Reinigung der Gay-Lussacsäure von Eisensalzen usw. kommt.

Um dieselbe Produktion zu erzwingen wie $\mathrm{N}$ i e d e $\mathbf{n} \mathfrak{f} \ddot{\mathbf{u}} \mathbf{h} \mathbf{r}$, bedarf es keineswegs der Gloverteilung, des Arbeitens mit außergewöhnlichem Überdruck, wie aus Faldings und seinen eigenen Mitteilungen hervorgeht.. Auch ohne die kostspielige Gloverteilung können andere in rationeller Anwendung all' der modernen Hilfsmittel und Erkenntnisse in bezug auf Kammer- und Turmdimensionierung und Gasführung dieselben Resultate er. zielen. 